\title{
Nonlinear System Identification Based on Reduced Complexity Volterra Models
}

\author{
Guodong $\operatorname{Jin}^{1, \mathrm{a}^{*}}$ and Libin Lu $\mathrm{u}^{1, \mathrm{~b}}$ \\ ${ }^{1}$ Xi'an research institute of high technology, Xi'an, 710025, P. R. China \\ ajinguodong_army@163.com, blulibin@126.com
}

Keywords: Nonlinear system; Volterra series model; Nonparametric model identification; Random multi-tone excitation

\begin{abstract}
Conventional Volterra series model is hardly applied to engineering practice due to its parametric complexity and estimation difficulty. To solve this problem, nonlinear system identification using reduced complexity Volterra models is proposed. Since the nonlinear components often play a secondary role compared to the dominant, linear component of the system, they spend the most of identification cost. So it is worth establishing a balance between identification cost and model accuracy by reducing the complexity of nonlinear components. Refer to the idea of nonlinear output frequency response function, conventional Volterra model is simplified. And then a minimum mean square error criterion based method to identify the simplified model is proposed. The distinguishing feature of this method is high accuracy, good robustness, and significant reduction in the computational requirements compare to the identification of conventional Volterra models. The simulation show that the proposed method is effective, and the reduced complexity Volterra model is of good generalization ability in general. So this nonlinear system identification approach is quite applicable to engineering practice.
\end{abstract}

\section{Introduction}

The Volterra series model provides an intuitive and relatively general framework for analyzing the behavior of non-linear systems [1]. Most real world systems are nonlinear in nature so thatnonlinear models are often preferable for representing systems under study. This is evidenced by the wild spread applications of Volterra models in fields including control [2], identification [3], damage detection [4] and assessment [5].

The objective of present study is to develop an efficient and practical approach for identification of quadratic nonlinear systems. A simplified nonparametric model identification method is presented. This method leads to significant reductions in both the computational requirements and the mathematical tractability comparing to traditional Volterra model. Simulations show present algorithm has excellent ability of model generalization and is still effective with output measurement noises, even in low signal-noise-ratio (SNR) condition.

\section{Preliminaries}

For a weakly nonlinear system up to second order Volterra series representation, the discrete time Volterra model can be expressed as

$$
y(k)=\sum_{n=1}^{\infty} \sum_{i_{1}=-\infty}^{\infty} \ldots \sum_{i_{n}=-\infty}^{\infty} h_{n}\left(i_{1}, \ldots, i_{n}\right) \prod_{i=1}^{n} u\left(t-i_{i}\right)
$$

where $h_{n}\left(\tau_{1}, \ldots, \tau_{n}\right)$ denote the nth-order Volterra kernel function. In the frequency domain, Eq.1 transforms to

$$
Y(f)=H_{1}(f) U(f)+\sum_{k} H_{2}(f-g, g) U(f-g) U(g)
$$

where $Y(f), U(f)$ denote the Fourier transforms of the output and input response, respectively. $H_{n}\left(f_{1}, \cdots, f_{n}\right)$ is the n-dimensional Fourier transform of $h_{n}\left(\tau_{1}, \ldots, \tau_{n}\right)$, which called the nth order GFRF: 


$$
H_{n}\left(f_{1}, \cdots, f_{n}\right)=\int_{-\infty}^{\infty} \cdots \int_{-\infty}^{\infty} h_{n}\left(\tau_{1}, \cdots, \tau_{n}\right) \exp \left(-j\left(2 \pi f_{1} \tau_{1}+\cdots+2 \pi f_{n} \tau_{n}\right)\right) d \tau_{1} \ldots d \tau_{n}
$$

The Eq.3 can be written more compactly with the linear and quadratic operators $\mathrm{L}($.$) and \mathrm{Q}($.$) :$

$$
Y=L(U)+Q(U)+\varepsilon
$$

where $\varepsilon$ denotes the model error in frequency domain. For practical reason, we consider input signals having negligible power at the frequencies larger than some upper bound frequency $f M$. Correspondingly the frequency ranges of each order outputs are $f \in\left[0, f_{M}\right]$ for linear part and $f$, $g \in\left[-f_{M}, f_{M}\right]$ or quadratic part. Therefore the valid frequency range of quadratic kernels is $\left[0,2 f_{M}\right]$ with the assumption of the GFRFs are symmetric without loss of generality.

\section{A Simplified Identification Method}

Consider the input and output signals are sampled synchronously with the sampling frequency fs . When the expected discrete frequency resolution $0 \mathrm{f}$ and the maximum input frequency $\mathrm{fM}=\mathrm{Mf0}$ are given, the least data length $\mathrm{N}$ should be taken as $4 \mathrm{M}$ to avoid aliasing. Then the quadratic Volterra model in the discrete frequency domain may be written as

$$
Y\left(k f_{0}\right)=H_{1}\left(k f_{0}\right) U\left(k f_{0}\right)+\sum_{m} H_{2}\left(m f_{0},(k-m) f_{0}\right) U\left((k-m) f_{0}\right)+\varepsilon\left(k f_{0}\right)
$$

for $k, m \in(-N / 2, N / 2]$. Since the higher order kernels often play a secondary role compared to the dominant, linear component of the system, it is reasonable to assume that all $H_{n}\left(f_{1}, \cdots, f_{n}\right)$ in the hyper plane $f_{1}+\ldots+f_{n}=f$ are the same value. Under this assumption, a conception of equivalent kernel is defined as

$$
H_{n}^{\text {equ }}\left(k f_{0}\right)=\frac{1}{(2 M)^{n-1}} \sum_{k_{1}+\ldots+k_{n}=k} H_{n}\left(k_{1} f_{0}, \ldots, k_{n} f_{0}\right)
$$

Therefore, the Eq.5 may be simplified to

$$
Y\left(k f_{0}\right)=H_{1}\left(k f_{0}\right) U\left(k f_{0}\right)+H_{2}^{e q u}\left(k f_{0}\right) \sum_{m} U\left(m f_{0}\right) U\left((k-m) f_{0}\right)+\varepsilon\left(k f_{0}\right)
$$

The unknown kernels in Eq.7 can be calculated approximately from the LSM (Least-SquareMinimization) of the power of model error. Define the least square cost function

$$
J=E\left\{\varepsilon^{2}\right\}=E\left\{(Y-L(U)-Q(U))^{2}\right\}
$$

Where $\mathrm{E}\{$.$\} denotes the expectation operator. To minimize Eq. 8$ requires

$$
\frac{\partial J}{\partial H_{1}}=\frac{\partial J}{\partial H_{2}^{\text {equ }}}=0
$$

Substituting Eq.8 into Eq.9, we obtain

$$
\begin{aligned}
\mathbf{Z} & =\boldsymbol{\Phi}^{T} \mathbf{H} \\
\mathbf{Z} & =\left[\begin{array}{lll}
S_{U Y}\left(k f_{0}\right) \ldots S_{U U Y}^{*}\left(g f_{0},(k-g) f_{0}\right) \ldots
\end{array}\right]^{T} \\
\mathbf{H} & =\left[\begin{array}{llll}
H_{1}\left(k f_{0}\right) & H_{2}^{e q u}\left(k f_{0}\right)
\end{array}\right]^{T} \\
\mathbf{\Phi} & =\left[\begin{array}{cccc}
S_{U U}\left(k f_{0}\right) & \ldots & S_{U U U}^{*}\left(g f_{0},(k-g) f\right) & \ldots \\
\sum_{g} S_{U U U}\left(g f_{0},(k-g) f_{0}\right) & \ldots & 2 S_{U U}\left(g f_{0}\right) S_{U U}((k-g) f) & \ldots
\end{array}\right]
\end{aligned}
$$

for $k \in[0,2 M], g \in[(k+1) / 2, M]$ when $\mathrm{k}$ is odd and $g \in[k / 2, M]$ when $\mathrm{k}$ is even. In Eq.10-Eq.13, $S_{U U}, S_{U Y}, S_{U U U}$ and $S_{U U Y}$ are the auto spectrum, cross spectrum, auto bi-spectrum and cross bi-spectrum, respectively, and $[.]^{T}$ denotes the transpose operator. Eq.10 is derived by assuming the fourth order cumulant function of input signal vanishes.

The Least-Square solution of Eq.10 is $(\Phi \Phi T)-1 \Phi Z$. By using the equivalent kernel, the size ofФФТ is reduced from $(\mathrm{M}-\mathrm{k} / 2+1) \times(\mathrm{M}-\mathrm{k} / 2+1)$ to $2 \times 2$, which implies the significant reduction of computational requirements and store spaces in solving Eq.10. 


\section{Identification Algorithm Using Random Multi-tone Excitation}

For given discrete frequency resolution f0 and the maximum input frequency Mf0 , a random multi-tone excitation signal could be designed as follow

$$
u(t)=\sum_{m=0}^{M} A_{m} \sin \left(2 \pi m f_{0}+\phi_{m}\right)
$$

where $\left\{A_{m}\right\}$ is the constant amplitudes, and $\{\phi m\}$ is the random phases which are independent, uniformly distributed random variables on $[0,2 \pi)$. It is proved that the fourth order cumulants of such random multi-tone signals fall faster to zero than the third order cumulants [6]. Thus we canassume that the inputs have a vanishing tri-spectrum, and therefore Eq.10 is valid.

The steps of the identification algorithm can be described as follow:

Step 1: Choose the frequency resolution f0and the maximum input frequency Mf0 . Generate M random numbers independently for $\{\phi \mathrm{m}\}$. Then construct the input signal by Eq.14.

Step 2: Determine the sampling frequency $\mathrm{f} s$ and the record length $\mathrm{N}$ follow the principle $\mathrm{f} s=\mathrm{N}$ f0 $\geqslant$ Mf0.

Step 3: Apply the excitation to the real process, and sample the input and output signals synchronously.

Step 4: Estimate the spectrums in Eq.11-Eq.13, and then obtain the estimates of $\mathrm{H} 1$ and $H_{2}^{\text {equ }}$ according to the Eq.10 solution.

Step 5: Test the generalization ability for the obtained model. If the result reaches required precision, end the algorithm, if not, turn back to step 1.

\section{Simulations}

In this section, simulations are provide using the system $y=-15 y-0.1 y 2+u+0.1 u 2$. Input signal for testing the generalization ability of model is selected as $\mathrm{u}(\mathrm{t})=2 \sin (20.25 \mathrm{t})+2 \sin (15.34 \mathrm{t})$, which is not used in identification.

In order to demonstrate the effects of the identification algorithm presented in this study, two groups of numerical simulation are conducted. One compares the identification performances using random inputs with using random multi-tone inputs. Another examines the effects of additive noise in output measurement. In latter simulations, performances between the represented method and the previous method in [7] are compared. The results are given in Fig. 1-Fig. 3.
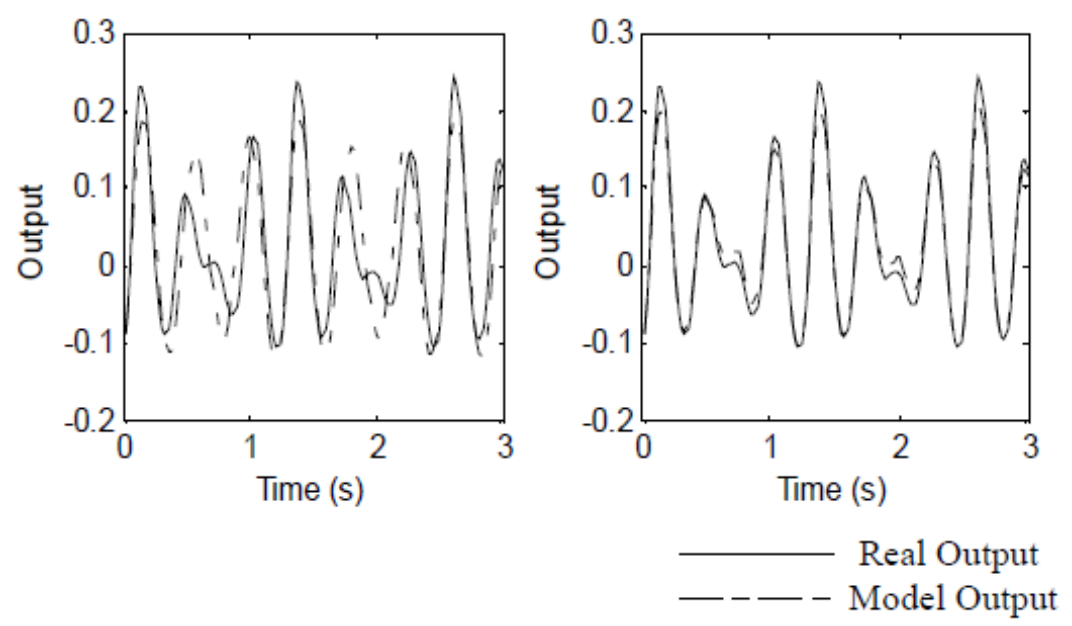

Figure 1. Output of identified model using random (left) and random multi-tone (right) inputs 

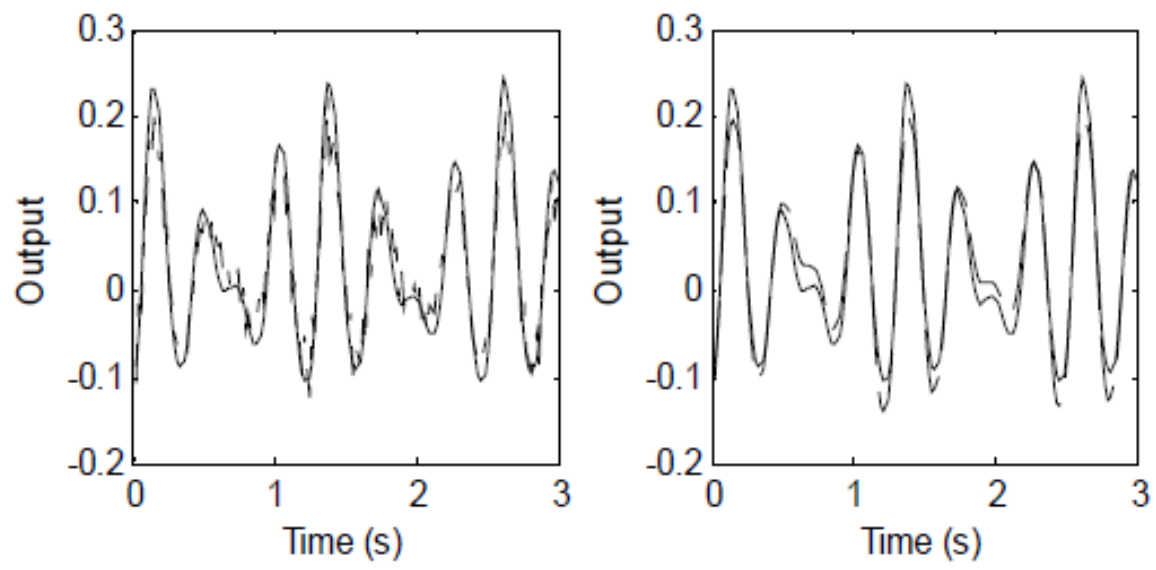

Figure 2. Output of identified model using previous (left) and present (right) methods (SNR: 20dB)
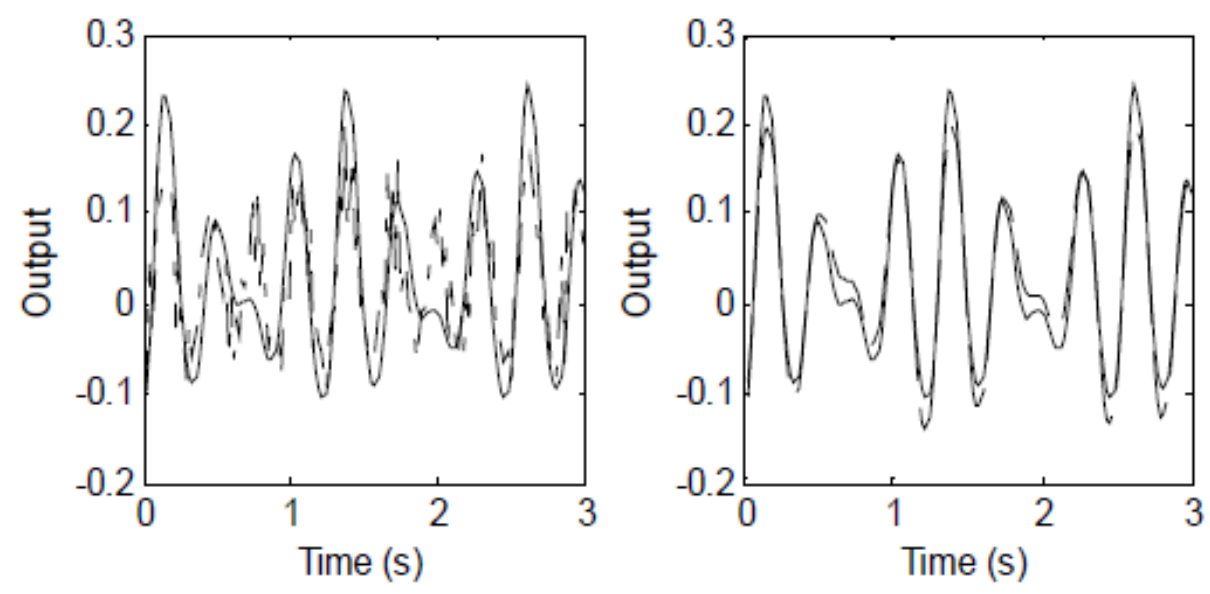

Figure 3. Output of identified model using previous (left) and present (right) methods (SNR: $10 \mathrm{~dB})$

In Fig. 1, we compare the performances of identified model using random and random multi-tone inputs. As can be seen in this figure, it is difficult to obtain accurate estimation using random inputs. For it is impossible to generate random inputs with perfect cut-off in its power spectrum, the accuracy of the estimation using random input is certainly affected. On the other hand, using random multi-tone inputs recover an excellent performance.

Fig. 2 and Fig. 3 compare the previous method and present method with additive noise in output measurement. It can be seen that the present method has a better ability of against the noise in output, which profit from the use of higher order spectrum. However, additive noise still result in inaccuracies in model identification, and by using more realizations, the error in kernel estimation may be decreased.

\section{Summary}

In this study, a simplified nonparametric identification algorithm for quadratic Volterra systems is proposed. By using multi-tone inputs and the higher order spectrum of input-output data, the generalization ability and the robustness of obtained model can be guaranteed. Moreover, compared to the previous methods, the present algorithm requires fewer calculations and store spaces.

\section{References}

[1] V. Volterra: Theory of Functionals and of Integral and Integro-differential Equations (Dover, New York 1959). 
[2] B.R. Maner, F.J. Doyle, B.A. Ogunnaike and R.K. Pearson: Nonlinear model predictive control of a simulated multivariable polymerization reactor using second-order Volterra models. Automatica, Vol. 32 (1996) No. 9, p.1285.

[3] C. Fernandes and G. Favier: Blind identification of multiuser nonlinear channels using tensor decomposition and precoding. Signal Processing, Vol. 89 (2009) No. 12, p.2644.

[4] I. Argatov and E.A. Butcher: On the separation of internal and boundary damage in slender bars using longitudinal vibration frequencies and equivalent linearization of damaged bolted joint response. Journal of Sound and Vibration, Vol. 330 (2011) No. 13, p.3245.

[5] A. Chatterjee: Structural damage assessment in a cantilever beam with a breathing crack using higher order frequency response functions. Journal of Sound and Vibration, Vol. 329 (2010) No.16, p.3325.

[6] A.M. Zoubir: Identification of Quadratic Volterra Systems Driven by Non-Gaussian Processes. IEEE Transaction on Signal Processing, Vol. 43 (1995) No. 5, p.1302.

[7] L.M. Li and S.A. Billings: Estimation of generalized frequency response functions for quadratically and cubically nonlinear systems. Journal of Sound and Vibration, Vol. 330 (2011), p.461.

[8] [M. Rebillat, R. Hennequin, E. Corteel, et al, "Identification of cascade of Hammerstein models for the description of non-linearities in vibrating devices", Journal of Sound and Vibration, Vol. 330, no. 5, pp.1018-1038, 2010.

[9] M. Schoukens, R. Pintelon, Y. Rolain, "Parametric identification of parallel Hammerstein systems", IEEE Transaction on Instrumentation and Measurement, Vol. 60, no. 12, pp.3931-3938, 2011.

[10]J. S. Bendat, Nonlinear System Analysis and Identification from Random Data, John Wiley \& Sons, New York, NY, 1990. 